\title{
Mortalidade por aborto no Brasil: Perfil e evolução de 2000 a 2020
}

\author{
Abortion mortality in Brazil: Profile and evolution from 2000 to 2020 \\ Mortalidad por aborto em Brasil: Perfil y evolución de 2000 a 2020
}

Vitoria Vilas Boas da Silva Bomfim ORCID: https://orcid.org/0000-0003-4897-0279

Centro Universitário Jorge Amado, Brasil E-mail: pesquisaclinica9@gmail.com

Maria Dhescyca Ingrid Silva Arruda ORCID: https://orcid.org/0000-0002-9073-7844

Faculdade São Francisco da Paraíba, Brasil E-mail: dhescycaingrid20@gmail.com

Emily da Silva Eberhardt

ORCID: https://orcid.org/0000-0003-2736-5686

Centro Universitário Cenecista de Osório, Brasil

E-mail: milyeberhardt@gmail.com

Nicoly Virgolino Caldeira

ORCID: https://orcid.org/0000-0002-7928-6333 Faculdade Santa Maria, Brasil E-mail: nicolyvirgulino14@gmail.com

Hozana Fernanda da Silva

ORCID: https://orcid.org/0000-0002-7210-4859 Universidade Maurício de Nassau, Brasil E-mail: hznfernanda@gmail.com

Angelica Ribeiro do Nascimento Oliveira

ORCID: https://orcid.org/0000-0002-7240-2004

Centro Universitário Maurício de Nassau, Brasil

E-mail: angelicalribeiro.ar19@gmail.com

Erlane Ribeiro dos Santos

ORCID: https://orcid.org/0000-0002-5889-8437 Universidade do Estado do Pará, Brasil

E-mail: erlanesantosribeirodossantos@gmail.com

Ludmilla Rafaela Marinho da Silva

ORCID: https://orcid.org/0000-0003-0025-2630

Pontifícia Universidade Católica de Minas Gerais, Brasil E-mail: ludmillarafaela30@gmail.com

Larissa Lima Soares

ORCID: https://orcid.org/0000-0002-7682-372X Centro Universitário Tiradentes, Brasil

E-mail: larissalyma@hotmail.com

Maria Eduarda Lopes de Macedo Bezerra

ORCID: https://orcid.org/0000-0001-6741-3190

Faculdade Estácio do Rio Grande do Norte, Brasil E-mail: eduardalopes022@gmail.com

Maiellen Pinheiro de Oliveira

ORCID: https://orcid.org/0000-0002-7235-6941

Centro Universitário Jorge Amado, Brasil E-mail: maiellen18@gmail.com

Gabriela Furlanetti de Pelegrini Freitas dos Anjos

ORCID: https://orcid.org/0000-0002-3125-8384

Universidade do Estado da Bahia, Brasil

E-mail: gabii.pelegrini@gmail.com

Renata Porangaba Cavalcante

ORCID: https://orcid.org/0000-0002-9408-3251

Centro Universitário Mario Pontes Juca, Brasil E-mail: porangabarenata@gmail.com

Priscila de Freitas Ferreira

ORCID: https://orcid.org/0000-0002-8716-7801

Universidade Federal de Juiz de Fora, Brasil E-mail: prifferreira84@gmail.com

João Felipe Tinto Silva

ORCID: https://orcid.org/0000-0003-3662-6673 


\begin{abstract}
Resumo
Objetivo: Identificar as desigualdades sociais e espaciais das mortes por aborto no Brasil, e simultaneamente, avaliar a evolução desses dados ao longo dos últimos anos. Métodos: Serão utilizados dados secundários sobre óbitos por abortos, registrados no SIM/SUS, no período de 2000 a 2020, para analisar os coeficientes de mortalidade por aborto no Brasil. Estes coeficientes serão calculados segundo variáveis relacionadas ao perfil sociodemográfico das mulheres que constam na base de dados do SIM/SUS, que constituem as variáveis de interesse do presente estudo. Resultados: Após levantamento de dados, foi possível perceber que a frequência entre as mulheres que mais morrem por aborto no Brasil, são mulheres em idade fértil de 20-29 anos, solteiras, e de cor parda. Considerações Finais: Constatou-se evidencias que provam o grande número de mulheres em idade fértil e com nível de escolaridade baixo, enfatizando a importância de implementar medidas de educação sexual e reforçar o planejamento familiar, a fim de evitar episódios de aborto e gestações indesejadas.
\end{abstract}

Palavras-chave: Sistemas de informação; Morte materna; Aborto; Saúde.

\begin{abstract}
Objective: To identify the social and spatial inequalities of abortion deaths in Brazil, and simultaneously assess the evolution of these data over the past few years. Methods: Secondary data on deaths from abortions, registered in the SIM/SUS, from 2000 to 2020, will be used to analyze the coefficients of mortality from abortion in Brazil. These coefficients will be calculated according to variables related to the sociodemographic profile of women in the SIM/SUS database, which constitute the variables of interest in this study. Results: After surveying data, it was possible to notice that the frequency among women who most die from abortion in Brazil are women of childbearing age 20-29 years old, single, and brown. Final Considerations: Evidence was found that proves the large number of women of childbearing age and with a low level of education, emphasizing the importance of implementing sexual education measures and strengthening family planning, in order to avoid episodes of abortion and unwanted pregnancies.
\end{abstract}

Keywords: Information systems; Maternal death; Abortion; Health.

\title{
Resumen
}

Objetivo: Identificar las desigualdades sociales y espaciales de las muertes por aborto en Brasil y, simultáneamente, evaluar la evolución de estos datos en los últimos años. Métodos: Los datos secundarios sobre muertes por aborto, registrados en el SIM / SUS, de 2000 a 2020, se utilizarán para analizar los coeficientes de mortalidad por aborto en Brasil. Estos coeficientes se calcularán en función de variables relacionadas con el perfil sociodemográfico de las mujeres en la base de datos SIM / SUS, que constituyen las variables de interés en este estudio. Resultados: Después de la encuesta de datos, se pudo notar que la frecuencia entre las mujeres que más mueren por aborto en Brasil son mujeres en edad fértil de 20 a 29 años, solteras y morenas. Consideraciones Finales: Se encontró evidencia que comprueba la gran cantidad de mujeres en edad fértil y con bajo nivel educativo, enfatizando la importancia de implementar medidas de educación sexual y fortalecer la planificación familiar, a fin de evitar episodios de aborto y embarazos no deseados.

Palabras clave: Sistemas de información; Muerte materna; Aborto; Salud.

\section{Introdução}

Segundo a OMS a definição de aborto é: um procedimento que implica no término da gestação. A palavra aborto vem do latim aboriri que significa: "separação do sítio adequado". Esse termo trata-se do produto da concepção eliminado da cavidade uterina ou abortado, enquanto o termo abortamento, mais amplamente aceito na área da saúde, diz respeito ao processo de ameaça à gravidez que pode culminar ou não na perda gestacional, onde o abortamento pode ser espontâneo ou provocado pela gestante. O aborto espontâneo é a interrupção da gestação que ocorre sem nenhuma intervenção externa e pode ser causado por doenças da gestante, por problemas genéticos do embrião ou por traumas, podendos esses serem físicos ou psíquicos. O termo aborto provocado, aborto induzido ou aborto voluntário, refere-se à interrupção da gravidez causada por uma intervenção externa e intencional (Adesse et al., 2016).

No Código Penal Brasileiro, consta que o aborto só não é penalizado em dois casos: aborto necessário (se não há outro meio de salvar a vida da gestante) e aborto humanitário (em gravidez resultante de estupro). Frente a esta situação, os abortos realizados atualmente no Brasil são feitos de forma clandestina, transformando o que seria um procedimento simples e de baixo risco, em uma situação de alto risco para uma parte significativa das mulheres que não podem pagar por uma intervenção 
em clinicas privadas, onde se tem acesso a abortos mais seguros. Lembrando que aborto é crime com pena prevista de um a três anos para as mulheres e de um a dez anos para quem executar o procedimento (Cook et al., 2004; Torres, 2012).

O Ministério da saúde criou uma norma técnica denominada "atenção humanizada ao abortamento" onde afirma que o abortamento representa no Brasil um sério problema de saúde pública por se tratar de uns pais em desenvolvimento onde se discutem complexos como, por exemplo: aspectos éticos, legais, morais, religiosos, culturais e até sociais acerca do assunto. Sabe-se que o abortamento é em sua maioria praticado, com o uso de meios diversos acarretando riscos e danos à saúde da mulher, muitas vezes induzidos pela própria ou realizados por profissionais em condições inseguras, sem preparo, capacitação e em geral provocando consequências danosas à saúde, podendo, inclusive, levar à morte (Domingues, 2008; Santos et al., 2013).

O perfil das mulheres que abortam, geralmente, é bem estabelecido, são predominantemente, mulheres entre 20 e 29 anos, em união estável, com até oito anos de estudo, trabalhadoras, católicas, com pelo menos um filho e usuárias de métodos contraceptivos, as quais abortam com misoprostol. As mesmas recorreram a técnicas inseguras, com chances de complicações imediatas que resultem no desfecho fatal. As complicações decorrentes dos abortos inseguros se constituem a terceira causa mais frequente de mortalidade materna (de Faro Sandi \& Braz, 2010).

As altas taxas de aborto, no Brasil, refletem as dificuldades enfrentadas pelas mulheres e seus parceiros diante das limitações de acesso a um planejamento familiar eficaz, que proporcione orientações e disponibilize métodos contraceptivos seguros. Este acesso é importante para reduzir os casos de gravidez indesejada e, consequentemente, a prática do aborto (Diniz et al., 2009).

Diversos estudos comprovam com a prática do aborto e suas eventuais complicações, no Brasil, estão intimamente relacionadas as desigualdades sociais existentes no país. Nos anos 90, registrou-se no Brasil uma redução da frequência de complicações por abortamento, atribuída ao uso do misoprostol pelas mulheres para autoindução do aborto, abandonando métodos mais agressivos utilizados na década de 80. A dificuldade da fala devido ao medo do crime, leva a subnotificação das mortes por aborto (de Mello \& Galli, 2016).

A mulher muitas vezes acaba abortando em sua própria residência e não procurando ajuda junto às equipes de saúde. Pode-se entender que por medo, dúvidas e até receio de julgamentos, por parte da equipe médica ou de familiares próximos. É de suma importância que Promoção do acolhimento, a informação, a orientação e o suporte emocional no atendimento dessas mulheres que favorece a atenção humanizada por meio da interação da equipe com a mulher, o que determina as percepções desta quanto à qualidade da assistência e do cuidado, melhorando a relação entre o profissional de saúde e usuária, aumenta a capacidade de resposta do serviço e o grau de satisfação das mulheres com o serviço prestado fazendo com que essa mulher perda o medo, assim a influência na decisão pela busca de um futuro atendimento. O profissional deve-se atentar nos casos de abortamento por estupro, onde o mesmo deverá atuar como facilitador do processo de tomada de decisão pela mulher, respeitando-a e acolhendo a escutando, evitando julgamentos, agindo de forma preconceituosa e até comentários desrespeitosos, deve agir com uma abordagem que respeite a autonomia das mulheres e seu poder de tomada de decisão, procurando estabelecer relação de confiança e cuidado (Borsari et al., 2012; Lopes \& Oliveira, 2020).

O presente trabalho pretende, portanto, apresentar um panorama atualizado das mortes por aborto no Brasil de 2000 a 2020, exatamente na última década onde o uso do misoprostol foi crescente no país. Além disso, se busca analisar a mortalidade por aborto segundo indicadores sociodemográficos das mulheres e regiões do país. Objetiva-se, desse modo, identificar as desigualdades sociais e espaciais das mortes por aborto no Brasil, e simultaneamente, avaliar a evolução desses dados ao longo dos últimos anos. 


\section{Metodologia}

Estudo transversal, de caráter analítico e abordagem quantitativa, o estudo quantitativo representa os dados por meio de variáveis estatísticas como valor real, medias, medianas e porcentagens, sendo dividido em três etapas coleta de dados, análise de dados e medidas estatísticas, apresentação dos dados em forma de resultados (Santos et al., 2017). O presente trabalho se propõe a analisar os coeficientes de mortalidade por aborto no Brasil, utilizando dados secundários sobre óbitos por abortos, registrados no Sistema de Informação sobre Mortalidade do Sistema Único de Saúde (SIM/SUS), no período de 2000 a 2020. Serão considerados óbitos por aborto aqueles classificados no capitulo XV da Classificação Internacional de Doenças vigente (CID 10), especificamente os itens O00-O08 (Gravidez que termina em aborto), entre mulheres em idade reprodutiva (10 a 49 anos). Os coeficientes da mortalidade por aborto serão calculados segundo variáveis relacionadas ao perfil sociodemográfico das mulheres (faixa etária, cor/raça, grau de escolaridade, estado civil e as cinco macrorregiões de residência - Norte, Sul, Sudeste, Centro-Oeste e Nordeste), variáveis que constam na base de dados do SIM/SUS e constituem-se de interesse para o presente estudo. Os dados sobre população serão obtidos na Fundação Instituto Brasileiro de Geografia e Estatística (IBGE) (Brasil, 2010; DATASUS, 2021). Para análise dos dados será utilizado o software Tabwin (versão 3.6) disponível no site do DATASUS.

\section{Resultados}

Após a aplicação da busca que fora realizada através do Sistema de Informação sobre Mortalidade (SIM), os dados foram trabalhados, exportados para o Excel $^{\circledR}$ (versão 2016) e posteriormente, exemplificados em forma de tabelas e mapa, os quais permitem uma boa visualização e análise das variáveis adotadas para o estudo. A partir dos dados extraídos pelo DataSUS, o mapa exemplifica os casos de aborto entre os anos de 2010 a 2019 no Brasil. De modo que, os estados com maiores índices de aborto nos últimos 10 anos foram São Paulo com o total de 133350 (20.27\%), Minas Gerais com 66649 (10.13\%) e Rio de Janeiro que teve 66269 (10.07\%) casos de abortos (DATASUS, 2021).

Figura 1 - Mapa de casos de aborto de 2010 a 2019 no Brasil. Brasil, 2021.

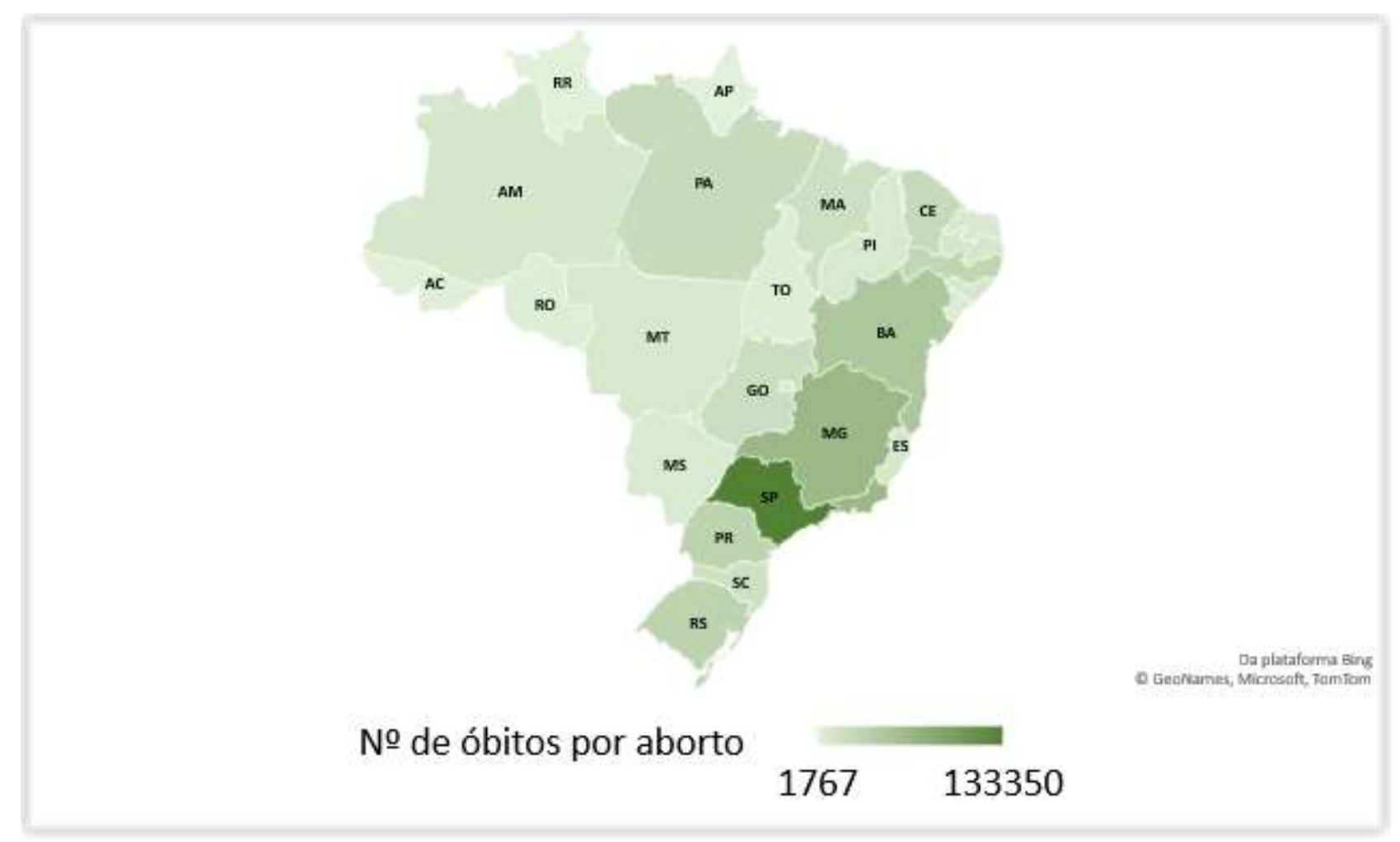




\section{Fonte: Autores via DATASUS (2021).}

Foi realizado o cálculo de porcentagem de cada variável, considerando: faixa etária, cor/raça, grau de escolaridade e estado civil. A coleta foi realizada através da utilização do CID10 [O00 - O08], o resultado de maior porcentagem foram mulheres com idade de 20 a 29 anos, com $44.70 \%$, seguido por mulheres de 30 a 39 anos com taxa 33.17\%, logo seguido por jovens de 15-19 anos aparecendo 14.71\%, percebe-se também a faixa etária de 40 aos 49 anos com 6.38\% de casos registrados, como contraste é possível observar que a menor porcentagem é representada por $1.04 \%$, caracterizada por mulheres de 10 a 14 anos (Tabela 1) (DATASUS, 2021).

Tabela 1 - Caracterização das amostras por faixa etária no ano de 2020. Brasil, 2021.

\begin{tabular}{ccc}
\hline IDADE POR FAIXA ETÁRIA (ANOS) & N & $\%$ \\
\hline $10-14$ & 28 & 1.04 \\
$15-19$ & 397 & 14.71 \\
$20-29$ & 1206 & 44.70 \\
$30-39$ & 895 & 33.17 \\
$40-49$ & 172 & 6.38 \\
\hline Total & 2698 & 100 \\
\hline
\end{tabular}

Fonte: Autores via DATASUS (2021).

Quando analisado a variável raça/cor que segundo o IBGE (2010) são pessoas que se autodeclaram como brancas, pretas, amarelas, pardas e indígenas. Dentre estas, destacam-se as com maior porcentagem sendo, mulheres da cor parda com 48.59\%, em seguida as brancas com 31.80, pode-se observar ainda que as mulheres negras representam $11.60 \%$ dos casos, como podese observar na Tabela 2 (DATASUS, 2021).

Tabela 2 - Caracterização das amostras segundo cor/raça no ano de 2020. Brasil, 2021.

\begin{tabular}{ccc}
\hline COR/RAÇA & N & $\%$ \\
Branca & 858 & 31.80 \\
Preta & 313 & 11.60 \\
Amarela & 5 & 0.18 \\
Parda & 1311 & 48.59 \\
Indígena & 34 & 1.26 \\
Ignorado & 177 & 6.56 \\
\hline Total & 2698 & 100 \\
\hline
\end{tabular}

Fonte: Autores via DATASUS (2021). 
Sendo assim, em seguida foi possível verificar as variáveis segundo o grau de escolaridade das mulheres analisadas, dentre as quais é possível inferir que a maioria delas haviam cursado apenas o Ensino Fundamental, conforme mostra a porcentagem de 38.91\%, seguido com Ensino Médio representado por 26.72\%, sendo a menor parte o Ensino Superior com apenas 6\%, desconsiderando as escolaridades ignorada (Tabela 3) (DATASUS, 2021).

Tabela 3 - Caracterização das amostras segundo grau de escolaridade no ano de 2020. Brasil, 2021.

\begin{tabular}{lcc}
\hline \multicolumn{1}{c}{ GRAU DE ESCOLARIDADE } & $\mathrm{N}$ & $\%$ \\
\hline E. Fundamental & 1050 & 38.91 \\
E. Médio & 723 & 26.79 \\
E. Superior & 162 & 6.00 \\
Nenhuma & 96 & 3.55 \\
Ignorado & 667 & 24.72 \\
\hline Total & 2.698 & 100 \\
\hline
\end{tabular}

Fonte: Autores via DATASUS (2021).

Após o levantamento das variáveis segundo o estado civil, conforme a Tabela 4, foi constatado que a maioria das mulheres eram caracterizadas como solteiras representando $62.56 \%$ do total das analisadas, acompanhada pela variável do estado de casada com 20.53\%. Ademais, a menor parte das mulheres eram viúvas e separadas judicialmente (DATASUS, 2021).

Tabela 4 - Caracterização das amostras segundo estado civil no ano de 2020. Brasil, 2021.

\begin{tabular}{|c|c|c|}
\hline ESTADO CIVIL & $\mathbf{N}$ & $\%$ \\
\hline Solteira & 1688 & 62.56 \\
\hline Casada & 554 & 20.53 \\
\hline Viúvo & 28 & 1.03 \\
\hline Separado Judicialmente & 54 & 2.00 \\
\hline Outro & 163 & 6.04 \\
\hline Ignorado & 211 & 7.82 \\
\hline Total & 2698 & 100 \\
\hline
\end{tabular}

Fonte: Autores via DATASUS (2021).

\section{Discussão}

No Brasil, a questão do aborto é considerada uma adversidade de saúde pública, essa prática é descrita como a maior causa de morte materna, para classificar como óbito materno deve-se investigar a mulher em idade fértil (Cardoso et al., 2020). Sendo assim, a partir do levantamento de dados obteve-se que a taxa de aborto é maior entre mulheres de faixa etária 
entre 20-29 anos, o que evidencia uma falta na inserção junto com os parceiros na busca de um planejamento familiar, possibilitando assim o rastreamento de possíveis complicações, contratempos que resultam em morte fetal e, consequentemente em impactos significativos na qualidade de vida da mulher (Menezes et al., 2020).

Segundo dados coletados no DataSUS, O Brasil chega a registar diariamente 535 internações causadas por aborto. Verificou-se que em 2019, a cada 100 internações 99 foram causadas por aborto espontâneos do tipo de causas indeterminadas e apenas 1 de aborto previsto em lei, como em casos de estupro ou risco a vida da gestante. Percebeu-se ainda, que as principais vítimas de procedimentos de aborto em geral são mulheres negras que cada vez são mulheres mais jovens. Entre os anos de 2009 a 2018, o Sistema Único de Saúde (SUS) registrou oficialmente 721 mortes de mulheres por aborto em sua maioria negras ou pardas com baixo acesso a informações e cuidados humanizados. A cada 10 mulheres que vinham a óbito, 6 eram pretas ou pardas. Ressalta-se ainda que apenas de 2010 a 2019, o SUS registrou 24,8 mil internações por aborto de meninas de 10 até 14 anos números alarmantes em uns pais em desenvolvimento. Lembra-se que a maioria das ocorrências dos abortos em meninas de 10 até 14 anos acontece no Nordeste por se tratar da região mais pobre do país com baixa acessibilidade a recursos pagos e até mesmo informações acerca do assunto (DATASUS, 2021).

Quando falamos em desigualdades, não se pode deixar de fora o grau de escolaridade, pois este quesito entra como fatores que induzem na ocorrência dos casos de aborto no Brasil, devido à falta de acesso à informações, principalmente no que se refere sobre educação sexual e planejamento familiar, ou seja, há uma deficiência de acompanhamento adequado para prevenção desses casos e de informações necessárias para esquivar-se dos números elevados de aborto (Anjos, Santos, Souzas, \& Eugênio, 2013). A partir disso, foi possível verificar que as mulheres com menor nível de escolaridade segundo os dados do DATASUS (Tabela 3) foram as maiores vítimas do aborto clandestino e que representaram um total de $38.91 \%$, evidenciando como a falta de informação sobre os riscos desse tipo de procedimento podem ser nocivos (Santos \& Silveira, 2017).

Dentre os principais fatores que estão ligados aos casos de aborto por causas não genéticas, há uma maior predominância em mulheres solteiras pelo fato de não utilizarem os métodos contraceptivos, acarretando em uma gravidez indesejada, que acabam gerando episódios de aborto e muitas vezes as mesmas possuem múltiplos parceiros que resultam também como um fator que influencia para realizar o ato abortivo, pois, a falta de apoio sentimental de um parceiro pode ser um fator ligado diretamente a decisão do abortamento, haja vista que a mulher poder estar com medo e confusa no momento em que descobre a gravidez (Sell et al., 2015; Ribeiro \& Spink, 2011).

Em mulheres pardas a taxa é de $48,59 \%$, não sendo muito distante de mulheres declaradas brancas as quais representam $31.80 \%$, sendo estas as taxas mais altas segundo a tabela 2 , apontando uma elevação nos casos no que se refere à cor/raça, sendo este interligado com a falta de planejamento familiar, e consequentemente sobre conhecimento dos métodos contraceptivos, e que alguns desses são fornecidos gratuitamente pelo governo, demonstrando muitas vezes que a baixa escolaridade impacta diretamente na vida da mulher, pois, novamente, evidencia a falta de democratização a informação. Vale ressaltar que a prática do aborto pode causar sequelas significativas na saúde mental e física da mulher, podendo progredir para quadros hemorrágicos e infecciosos graves, quando se trata da saúde física, e casos de depressão e ansiedade, se tratando da saúde psicológica das mesmas, quando realizados de maneira clandestina, visto que o aborto não é uma prática legalizada no Brasil e sendo assim, não possuem qualquer planejamento de acolhimento para essas mulheres (DATASUS, 2021; Scavone, 2008; Pereira et al., 2017).

\section{Considerações Finais}

O presente estudo alcançou o objetivo que foi identificar as desigualdades sociais e espaciais das mortes por aborto no Brasil e avaliar a evolução desses dados ao longo dos últimos anos. Apesar de não haver dentro do Sistema de Informação, as causas por aborto inseguro, o que não fortalece propriamente dito os números brutos de casos no Brasil, ainda há um aumento 
significativos de morte materna por aborto, por isto, constatou-se evidencias que provam o grande número de mulheres em idade fértil e com nível de escolaridade baixo, enfatizando a importância de implementar medidas de educação sexual e reforçar o planejamento familiar, a fim de evitar episódios de aborto e gestações indesejadas. Deste modo, este estudo aponta para a necessidade de mais pesquisas sobre a temática, com o intuito de se obterem retratos mais apurados, dos casos de morte por aborto no Brasil, tais como as causas por aborto inseguro, a fim de estimular a criação de políticas públicas que alberguem os determinantes sociais que envolvem o aborto no país.

\section{Referências}

Adesse, L., Jannotti, C. B., Silva, K. S. D., \& Fonseca, V. M. (2016). Aborto e estigma: uma análise da produção científica sobre a temática. Ciência \& Saúde Coletiva, 21, 3819-3832.

Anjos, K. F. D., Santos, V. C., Souzas, R., \& Eugênio, B. G. (2013). Aborto e saúde pública no Brasil: reflexões sob a perspectiva dos direitos humanos. Saúde em Debate, 37, 504-515.

Borsari, C. M. G., Nomura, R. M. Y., Benute, G. G., Nonnenmacher, D., Lucia, M. C. S. D., \& Francisco, R. P. V. (2012). O aborto inseguro é um problema de saúde pública. Femina.

Brasil (2010). Censo demográfico IBGE 2010: características gerais da população. http://censo2010.ibge.gov.br/

Cardoso, B. B., Vieira, F. M. D. S. B., \& Saraceni, V. (2020). Aborto no Brasil: o que dizem os dados oficiais? Cadernos de Saúde Pública, 36 , e00188718.

Cook, R. J., Dickens, B. M., \& Fathalla, M. F. (2004). Saúde reprodutiva e direitos humanos. Integrando medicina, ética e direito. Tradução Andrea Romani e Renata Perrone e equipe. Rio de Janeiro: Cepia.

DATASUS. (2021). Sistema de Informação sobre Mortalidade do Sistema Único de Saúde (SIM/SUS). http://www2.datasus.gov.br/DATASUS/index.php?area=060701

de Faro Sandi, S., \& Braz, M. (2010). As mulheres brasileiras e o aborto: uma abordagem bioética na saúde pública. Revista Bioética, 18(1).

de Mello, M. E. V., \& Galli, B. (2016). Mortalidade Materna e Aborto Inseguro: uma questão de direitos humanos das mulheres.

Diniz, D., Corrêa, M., Squinca, F., \& Braga, K. S. (2009). Aborto: 20 anos de pesquisas no Brasil. Cadernos de Saúde Pública, 25(4), 939-942.

Domingues, R. C. (2008). Entre normas e fatos, o direto de decidir: o debate sobre o aborto à luz dos princípios constitucionais. Direito de decidir: múltiplos olhares sobre o aborto. Belo Horizonte: Autêntica, 67-103.

Lopes, S. D. N., \& Oliveira, M. H. B. D. (2020). Meu corpo, minhas regras: mulheres na luta pelo acesso ao serviço público de saúde para a realização do aborto seguro. Saúde em Debate, 43, 20-33.

Menezes, G., Aquino, E. M., Fonseca, S. C., \& Domingues, R. M. S. M. (2020). Aborto e saúde no Brasil: desafios para a pesqui sa sobre o tema em um contexto de ilegalidade. Cadernos de Saúde Pública, 36, e00197918.

Pereira, L. J. E., da Nóbrega, T. B. T., \& Almeida, F. L. (2017). A Criminalização Do Aborto Como Uma Expressão De Subjetividade Do Sofrimento Feminino. https://editorarealize.com.br/editora/anais/conages/2018/TRABALHO_EV112_MD1_SA11_ID137_09052018212725.pdf

Ribeiro, F. R. G., \& Spink, M. J. P. (2011). Repertórios interpretativos na controvérsia sobre a legalização do aborto de fetos anencefálicos. Psicologia \& Sociedade, 23(spe), 63-71.

Santos, C. S., \& Silveira, L. M. C. D. (2017). Percepções de mulheres que vivenciaram o aborto sobre autonomia do corpo feminino. Psicologia: Ciência e Profissão, 37(2), 304-317.

Santos, J. L. G. D., Erdmann, A. L., Meirelles, B. H. S., Lanzoni, G. M. D. M., Cunha, V. P. D., \& Ross, R. (2017). Integração entre dados quantitativos e qualitativos em uma pesquisa de métodos mistos. Texto \& Contexto-Enfermagem, 26(3).

Santos, V. C., Anjos, K. F. D., Souzas, R., \& Eugênio, B. G. (2013). Criminalização do aborto no Brasil e implicações à saúde pública. Revista Bioética, 21(3), 494-508.

Scavone, L. (2008). Políticas feministas do aborto. Revista Estudos Feministas, 16(2), 675-680.

Sell, S. E., dos Santos, E. K. A., Velho, M. B., Erdmann, A. L., \& Rodriguez, M. D. J. H. (2015). Motivos e significados atribuídos pelas mulheres que vivenciaram o aborto induzido: revisão integrativa. Revista da Escola de Enfermagem da USP, 49(3), 495-501.

Torres, J. H. R. (2012). Aborto e legislação comparada. Ciência e cultura, 64(2), 40-44. 